\title{
Mining Sectorial Episodes from Event Sequences
}

\author{
Takashi Katoh ${ }^{1}$, Kouichi Hirata ${ }^{2}$, and Masateru Harao ${ }^{2}$ \\ 1 Graduate School of Computer Science and Systems Engineering \\ 2 Department of Artificial Intelligence \\ Kyushu Institute of Technology \\ Kawazu 680-4, Iizuka 820-8502, Japan \\ t_katou@dumbo.ai.kyutech.ac.jp \\ \{hirata, harao\}@ai.kyutech.ac.jp
}

\begin{abstract}
In this paper, we introduce a sectorial episode of the form $C \mapsto r$, where $C$ is a set of events and $r$ is an event. The sectorial episode $C \mapsto r$ means that every event of $C$ is followed by an event $r$. Then, by formulating the support and the confidence of sectorial episodes, in this paper, we design the algorithm SECT to extract all of the sectorial episodes that are frequent and confidential from a given event sequence by traversing it just once. Finally, by applying the algorithm SECT to bacterial culture data, we extract sectorial episodes representing $d r u g$ resistant change.
\end{abstract}

\section{Introduction}

The sequential pattern mining $[3,6,7,9,10]$ is one of the data mining methods from time-related data. The purpose of sequential pattern mining is to discover frequent subsequences as patterns in a sequential database. On the other hand, the episode mining $[4,5]$ introduced by Mannila et al. [5] is known as another approach to discover frequent patterns from time-related data. The purpose of episode mining is to discover frequent episodes, not subsequences, that are a collection of events occurring frequently together in event sequences.

In episode mining, the frequency is formulated as the number of occurrences of episodes in every window that is a subsequence of event sequences under a fixed time span called the width of windows. Then, Mannila et al. [5] have introduced a parallel episode as a set of events and a serial episode as a sequence of events. By combining the above episodes, they have extended the forms of episodes as directed acyclic graphs of events of which edges specify the temporal precedentsubsequent relationship.

Concerned with episode mining, in this paper, we introduce a sectorial episode of the form $C \mapsto r$, where $C$ is a parallel episode and $r$ is an event. The sectorial episode $C \mapsto r$ means that every event of $C$ is followed by an event $r$, so we can regard every event in $C$ as a causation of $r$. Note that, since a sectorial episode is captured as the direct precedent-subsequent relationship between events, it is just a candidate of causality in database ( $c f$. [11]).

We formulate the support $\operatorname{supp}(C \mapsto r)$ of a sectorial episode $C \mapsto r$ as the ratio of the number of $k$-windows (i.e., a window with width $k$ ) in which $C \mapsto r$ 
occurs for the number of all $k$-windows. For the minimum support $\sigma$ such that $0<\sigma<1$, we say that $C \mapsto r$ is frequent if $\operatorname{supp}(C \mapsto r) \geq \sigma$. Also we can show that the sectorial episode preserves anti-monotonicity, that is, for $C_{1} \subseteq C_{2}$, if $C_{2} \mapsto r$ is frequent then so is $C_{1} \mapsto r$. Furthermore, by regarding a sectorial episode as an association rule $[1,2]$, we introduce the confidence conf $(C \mapsto r)$ of a sectorial episode $C \mapsto r$ as the ratio of the number of $k$-windows in which $C \mapsto r$ occurs for the number of all $k$-windows in which $C$ occurs. For the minimum confidence $\gamma$ such that $0<\gamma<1$, we say that $C \mapsto r$ is confidential if $\operatorname{conf}(C \mapsto r) \geq \gamma$.

The purpose of this paper is to design the algorithm to extract all of the sectorial episodes that are frequent and confidential from a given event sequence. Since our sectorial episode is a combination of parallel and serial episodes, it is possible to extract all of the sectorial episodes that are frequent and confidential by combining the algorithms designed in [5]. On the other hand, in this paper, we design another algorithm SECT to extract them efficiently and appropriate to sectorial episodes.

The algorithm SECT consists of the following two procedures. The first procedure SCAN is to extract all of the frequent sectorial episodes of the form $C \mapsto r$ such that $|C|=1$ and then store the information of windows in which $C \mapsto r$ occurs. The second procedure is to extract all of the frequent sectorial episodes of the form $C \mapsto r$ such that $|C| \geq 2$ for every event $r$ stored by Scan. Here, the frequency of the constructed sectorial episodes is computed by using the anti-monotonicity, as similar as the well-known algorithm APRIORITID [1,2].

Then, we show that the algorithm SCAN runs in $O\left((l+k)|\mathcal{E}|^{2}\right)$ time and space, where $l$ is the time span between the starting time and the ending time in a given event sequence, $k$ is the width of windows and $\mathcal{E}$ is a set of all events, by traversing the event sequence just once. Hence, we show that the algorithm SECT extracts all of the sectorial episodes that are frequent and confidential from the sectorial episodes by SCAN in $O\left((l+k)|\mathcal{E}|^{2 M+1}\right)$ time and $O\left((l+k)|\mathcal{E}|^{M}\right)$ space without traversing the event sequence, where $M=\max \{|C| \mid C \mapsto r$ is frequent $\}$.

Finally, we apply the algorithm SECT to bacterial culture data. Note that, from the medical viewpoint, in order to extract sectorial episodes concerned with drug-resistant change, it is necessary to extract them based on the same detected bacterium and the same sample. Hence, in this paper, we divide the database into pages for the detected bacterium and the sample in whole 44 attributes, and then extract sectorial episodes representing drug-resistant changes from them.

\section{Sectorial Episodes}

As similar as [5], we assume that an event has an associated time of occurrence as a natural number. Formally, let $\mathcal{E}$ be a set of event types. Then, a pair $(e, t)$ is called an event, where $e \in \mathcal{E}$ and $t$ is a natural number which is the (occurrence) time of the event. In the following, for a set $E \subseteq \mathcal{E}$ of event types, we denote $\{(e, t) \mid e \in \mathcal{E}\}$ by $(E, t)$, and also call it by an event again. Furthermore, we denote a set $\left\{e_{1}, \ldots, e_{m}\right\} \subseteq \mathcal{E}$ of event types by a string $e_{1} \cdots e_{m}$. 
An event sequence $\mathcal{S}$ on $\mathcal{E}$ is a triple $\left(S, T_{s}, T_{e}\right)$, where

$$
S=\left\langle\left(E_{1}, t_{1}\right), \ldots,\left(E_{n}, t_{n}\right)\right\rangle
$$

is an ordered sequence of events satisfying the following conditions.

1. $E_{i} \subseteq \mathcal{E}(1 \leq i \leq n)$,

2. $t_{i}<t_{i+1}(1 \leq i \leq n-1)$, and

3. $T_{s} \leq t_{i}<T_{e}(1 \leq i \leq n)$.

In particular, $T_{s}$ and $T_{e}$ are called the starting time and the ending time of $\mathcal{S}$. We denote $T_{e}-T_{s}$ by $l_{\mathcal{S}}$. For an event sequence $\mathcal{S}=\left(S, T_{s}, T_{e}\right)$, we denote the set of all event types of $\mathcal{S}$ at $t$, that is, $\{E \subseteq \mathcal{E} \mid(E, t) \in S\}$ by $\operatorname{evtyp}(\mathcal{S}, t)$.

A window in an event sequence $\mathcal{S}=\left(S, T_{s}, T_{e}\right)$ is an event sequence $W=$ $\left(w, t_{s}, t_{e}\right)$ such that $t_{s}<T_{e}, t_{e}>T_{s}$ and $w$ consists of all of the events $(E, t)$ in $S$ where $t_{s} \leq t<t_{e}$. The time span $t_{e}-t_{s}$ is called the width of the window. For a window $W=\left(w, t_{s}, t_{e}\right)$, we denote the starting time $t_{s}$ of $W$ by $\operatorname{st}(W)$ and the ending time $t_{e}$ of $W$ by et $(W)$.

We call a window with width $k$ in $\mathcal{S}$ a $k$-window, and denote the $k$-window $(w, t, t+k)$ of $\mathcal{S}$ starting from $t$ by $w(\mathcal{S}, t, k)$. For a $k$-window $W=w(\mathcal{S}, t, k)$, it is obvious that $s t(W)=t$ and $e t(W)=t+k$.

Note that we can regard a set $E=e_{1} \cdots e_{m}$ of event types as a parallel episode [5]. Here, we call the above $m$ the size of $E$. Furthermore, in this paper, we newly introduce the following sectorial episode.

Definition 1. Let $c_{1} \cdots c_{m} \subseteq \mathcal{E}$ be a parallel episode and $r$ an event type. Then, a sectorial episode is of the following form.

$$
X=c_{1} \cdots c_{m} \mapsto r .
$$

In this paper, we regard every event in $c_{1} \cdots c_{m}$ in a sectorial episode $X$ as a causation of $r$, so we call every $c_{i}$ and $r$ a causal type and a resulting type of $X$, respectively. For a set $C=c_{1} \cdots c_{m}$, we also denote $c_{1} \cdots c_{m} \mapsto r$ by $C \mapsto r$. In particular, we call a sectorial episode $C \mapsto r$ such that $r \in C$ trivial.

We call both a parallel episode and a sectorial episode episodes simply.

Definition 2. Let $\mathcal{S}$ be an event sequence $\mathcal{S}=\left(S, T_{s}, T_{e}\right)$ and $e$ an event type. Then, we say that $e$ occurs in $\mathcal{S}$ if there exists an event $(E, t) \in S$ such that $e \in E$. We denote $\{t \mid(E, t) \in S \wedge e \in E\}$ by $T(e, \mathcal{S})$. Also we denote $s t(e, \mathcal{S})=$ $\min \{t \mid t \in T(e, \mathcal{S})\}$ and $e t(e, \mathcal{S})=\max \{t \mid t \in T(e, \mathcal{S})\}$.

We say that a parallel episode $e_{1} \cdots e_{m}$ occurs in $\mathcal{S}$ if every $e_{i}$ occurs in $\mathcal{S}$. Also we say that a sectorial episode $c_{1} \cdots c_{m} \mapsto r$ occurs in $\mathcal{S}$ if $r$ occurs in $\mathcal{S}$, and, for every $i(1 \leq i \leq m), c_{i}$ occurs in $\mathcal{S}$ and $\operatorname{st}\left(c_{i}, \mathcal{S}\right)<e t(r, \mathcal{S})$.

Let $\mathcal{S}$ be an event sequence and $k$ a natural number. Then, we denote the set of all $k$-windows by $W(\mathcal{S}, k)$. Also, for an episode $X$, we denote the set of all $k$-windows such that $X$ occurs in $\mathcal{S}$ by $W(X, \mathcal{S}, k)$.

Note that we can number all $k$-windows in $W(\mathcal{S}, k)$ from $T_{s}-k$ to $T_{e}$. We call such a number $i\left(T_{s}-k<i<T_{e}\right)$ the label of the $i$-th $k$-window. For an event sequence $\mathcal{S}$ and an episode $X$, we identify $W(X, \mathcal{S}, k)$ with the set of all labels of $k$-windows in which $X$ occurs in $\mathcal{S}$. 


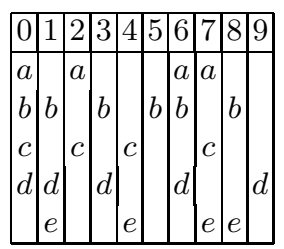

Fig. 1. An event sequence $S$ in Example 1.

Example 1. Let $\mathcal{E}=\{a, b, c, d, e\}$. Then, Figure 1 describes an event sequence $\mathcal{S}=(S, 0,10)$ on $\mathcal{E}$ where:

$$
\begin{gathered}
S=\langle(a b c d, 0),(b d e, 1),(a c, 2),(b d, 3),(c e, 4), \\
(b, 5),(a b d, 6),(a c e, 7),(b e, 8),(d, 9)\rangle .
\end{gathered}
$$

Furthermore, the event sequence $w=(\langle(a c, 2),(b d, 3),(c e, 4)\rangle, 2,5)$ is a 3 -window of $\mathcal{S}$ starting from 2, that is, $w=w(\mathcal{S}, 2,3)=(\langle(a c, 2),(b d, 3),(c e, 4)\rangle, 2,5)$.

For the above event sequence $\mathcal{S}$, it holds that $T(a, \mathcal{S})=\{1,2,6,7\}$, st $(a, \mathcal{S})=$ 1 and $e t(a, \mathcal{S})=7$. Also there exist 123 -windows, of which starting time is from -2 to 9 . Furthermore, for the above window $w$, sectorial episodes $a b \mapsto c, b c \mapsto e$ and $a c d \mapsto e$ occur in $w$, for example.

Let $\mathcal{S}$ be an event sequence, $X$ an episode and $k$ a natural number. Then, the frequency freq $_{\mathcal{S}, k}(X)$ and the support $\operatorname{supp}_{\mathcal{S}, k}(X)$ of $X$ in $\mathcal{S}$ w.r.t. $k$ are defined as follows.

$$
\operatorname{freq}_{\mathcal{S}, k}(X)=|W(X, \mathcal{S}, k)|, \operatorname{supp}_{\mathcal{S}, k}(X)=\frac{\operatorname{freq}_{\mathcal{S}, k}(X)}{|W(\mathcal{S}, k)|} .
$$

Definition 3. Let $\sigma$ be the minimum support such that $0<\sigma<1$. Then, we say that an episode $X$ is frequent if $\operatorname{supp}(X) \geq \sigma$.

Lemma 1 (Anti-monotonicity for sectorial episodes). Let $C_{1}$ and $C_{2}$ be parallel episodes such that $C_{1} \subseteq C_{2}$. If $C_{2} \mapsto r$ is frequent, then so is $C_{1} \mapsto r$.

Proof. It is sufficient to show that $W\left(C_{2} \mapsto r, \mathcal{S}, k\right) \subseteq W\left(C_{1} \mapsto r, \mathcal{S}, k\right)$. Suppose that $l \in W\left(C_{2} \mapsto r, \mathcal{S}, k\right)$ and let $W_{l}$ be the $l$-th $k$-window in $\mathcal{S}$. Then, it holds that $W_{l}=w\left(W\left(C_{2} \mapsto r, \mathcal{S}, k\right), l, k\right)$. For every $c \in C_{2}$, it holds that $\operatorname{st}\left(c, W_{i}\right)<$ et $\left(r, W_{l}\right)$. Since $C_{1} \subseteq C_{2}$, it holds that $s t\left(c^{\prime}, W_{i}\right)<e t\left(r, W_{l}\right)$ for every $c^{\prime} \in C_{1}$, so $C_{1} \mapsto r$ occurs in $W_{l}$. Hence, it holds that $l \in W\left(C_{1} \mapsto r, \mathcal{S}, k\right)$.

By regarding a sectorial episode $C \mapsto r$ as an association rule, we can introduce the confidence $\operatorname{conf}_{\mathcal{S}, k}(C \mapsto r)$ of $C \mapsto r$ in $\mathcal{S}$ w.r.t. $k$ as follows.

$$
\operatorname{conf}_{\mathcal{S}, k}(C \mapsto r)=\frac{|W(C \mapsto r, \mathcal{S}, k)|}{|W(C, \mathcal{S}, k)|} .
$$

Definition 4. Let $\gamma$ be the minimum confidence such that $0<\gamma<1$. Then, we say that a sectorial episode $C \mapsto r$ is confidential if $\operatorname{conf}(C \mapsto r) \geq \gamma$.

In the following, the subscripts $\mathcal{S}$ and $k$ in $\operatorname{freq}_{\mathcal{S}, k}(X), \operatorname{supp}_{\mathcal{S}, k}(X)$ and $\operatorname{conf} f_{\mathcal{S}, k}(X)$ are omitted, if they are clear by the context. 


\section{Algorithm to Extract Sectorial Episodes}

In this section, we design the algorithm to extract all of the sectorial episodes that are frequent and confidential from an event sequence $\mathcal{S}$, the minimum support $\sigma$, the minimum confidence $\gamma$, and the width $k$ of windows.

We assume the lexicographic order $\prec$ on $\mathcal{E}$. Also we extend $\prec$ to $2^{\mathcal{E}}$ as follows: For sets $Y=y_{1} \cdots y_{m}, Z=z_{1} \cdots z_{n}$ of event types, $Y \prec Z$ if there exists an $i$ $(1 \leq i \leq n)$ such that $y_{j}=z_{j}(1 \leq j \leq i-1)$ and $y_{i} \prec z_{i}$.

The algorithm SCAN described by Figure 2 extracts all of the frequent sectorial episodes of the form $c \mapsto r$, where $c$ and $r$ are events, and stores the set of labels of $k$-windows $W(c \mapsto r, \mathcal{S}, k)$ in which the sectorial episode $c \mapsto r$ occurs as $W[c][r]$ and the set of labels of $k$-windows $W(r, \mathcal{S}, k)$ for every $r \in \mathcal{E}$ as $V[r]$, by traversing $\mathcal{S}$ just once as similar as APRIORITID $[1,2]$.

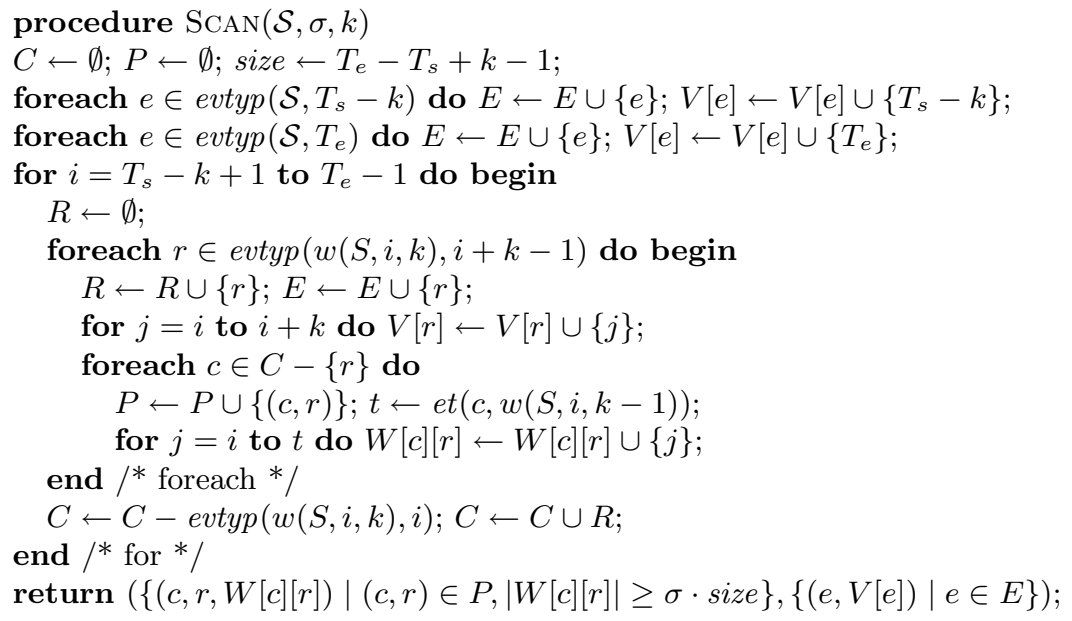

Fig. 2. The algorithm SCAN.

Lemma 2. Let $\mathcal{S}$ be an event sequence on $\mathcal{E}$ and $k$ the width of windows. Then, the algorithm SCAN extracts all of the frequent sectorial episodes of the form $c \mapsto r$ in $O\left(\left(l_{\mathcal{S}}+k\right)|\mathcal{E}|^{2}\right)$ time and space, by traversing $\mathcal{S}$ just once.

Proof. First, we show the correctness of the construction of $R$ and $C$. Fix an index $i\left(T_{s}+k-1 \leq i \leq T_{e}-1\right)$. Then, in the foreach-loop, the algorithm SCAN stores all elements in $\operatorname{evtyp}(w(S, i, k), i+k-1)$, where $\operatorname{et}(w(S, i, k))=i+k$, as $R$. Also it has stored all elements in $\bigcup_{j=i}^{i+k-1} \operatorname{evtyp}(w(S, i, k), j)$ as $C$.

Consider the case shifting $i$ to $i+1$. Since every element of $R$ becomes a causal type and every element of $\operatorname{evtyp}(w(S, i, k), i)-\bigcup_{j=i+1}^{i+k-1} \operatorname{evtyp}(w(S, i, k), j)$ does not 
become a causal type, the algorithm SCAN updates $C$ to $C-\operatorname{evtyp}(w(S, i, k), i)$ and then adds $R$ to $C$.

Next, for $r \in R$ and $c \in C-\{r\}$ on the $i$-th for-loop, consider the sectorial episode $c \mapsto r$. Let $t$ be $\operatorname{et}(c, w(S, i, k-1))$. Then, the causal type $c$ at $t$ is the nearest one to the resulting type $r$ in $w(S, i, k-1)$, so it holds that $c \mapsto r$ appears in the sequence $w(S, j, 1)$ of windows for $i \leq j \leq t$. Hence, by the inner for-loop, the algorithm SCAN adds the labels of such a sequence of windows to $W[c][r]$.

Hence, the algorithm SCAN stores all pairs $(c, r)$ such that $c \mapsto r$ appears in some $k$-window in $\mathcal{S}$ as $P$, so SCAN outputs the set of all frequent sectorial episodes of the form $c \mapsto r$.

Since the number of the outer for-loop is $T_{e}-T_{s}+k$ and the running time in the for-loop is $O\left(|\mathcal{E}|^{2}\right)$, the time complexity of the algorithm SCAN is $O(2|\mathcal{E}|+$ $\left.\left(T_{e}-T_{s}+k\right)|\mathcal{E}|^{2}+|\mathcal{E}|^{2}\right)=O\left(\left(l_{\mathcal{S}}+k\right)|\mathcal{E}|^{2}\right)$, where the first term $O(2|\mathcal{E}|)$ means the time of the first two foreach loops and the third term $O\left(|\mathcal{E}|^{2}\right)$ means the time checking frequency. Also the space of $W[c][r]$ and $V[e]$ are $O\left(\left(l_{\mathcal{S}}+k\right)|\mathcal{E}|^{2}\right)$ and $O\left(\left(l_{\mathcal{S}}+k\right)|\mathcal{E}|\right)$, respectively, so the space complexity of the algorithm SCAN is $O\left(\left(l_{\mathcal{S}}+k\right)|\mathcal{E}|^{2}\right)$. It is obvious that the algorithm SCAN traverses $\mathcal{S}$ just once.

After applying the algorithm SCAN, we apply the algorithm SECT described by Figure 3 to extract all of the sectorial episodes that are frequent and confidential. Here, the algorithm SECT extracts all frequent sectorial episodes $C \mapsto r$ where $|C| \geq 2$, by designing an APRIORITID-like procedure for every resulting type $r \in R[1,2]$. Here, the function $\operatorname{subs}(S, T)$ returns 1 if $S$ contains all of the subsets of $T$ of which length is $|T|-1$. For every resulting type $r \in R$, the algorithm SECT extracts all of the causal types $X c$ and stores them as $C[r]$ and $D[r]$, where $C[r]$ denotes the set of all causal types that $X c \mapsto r$ is frequent, and $D[r]$ denotes the set of all causal types that $X c \mapsto r$ is frequent and confidential.

The algorithm SECT computes the support of $X c \mapsto r$ by using $W(X, \mathcal{S}, k)$ and $W(c, \mathcal{S}, k)$. The following lemma guarantees the correctness of it.

Lemma 3. Let $C$ and $D$ be parallel episodes and $r$ an event type such that $r \notin C \cup D$. Then, the following statement holds:

1. $W(C \cap D, \mathcal{S}, k)=W(C, \mathcal{S}, k) \cap W(D, \mathcal{S}, k)$.

2. $W(C \cap D \mapsto r, \mathcal{S}, k)=W(C \mapsto r, \mathcal{S}, k) \cap W(D \mapsto r, \mathcal{S}, k)$.

Proof. The statement 1 is obvious. For the statement 2, suppose that $C=$ $c_{1} \cdots c_{m}$ and $D=d_{1} \cdots d_{n}$. Also let $l$ be a label such that $l \in W(C \cap D \mapsto$ $r, \mathcal{S}, k)$. Then, $C \cap D \mapsto r$ occurs in the $l$-th $k$-window $w(\mathcal{S}, l, k)$, so it holds that $\operatorname{st}\left(c_{i}, \mathcal{S}\right)<e t(r, \mathcal{S})$ and $\operatorname{st}\left(d_{j}, \mathcal{S}\right)<e t(r, \mathcal{S})$ for every $i$ and $j(1 \leq i \leq n$, $1 \leq j \leq m)$, which implies that $l \in W(C \mapsto r, \mathcal{S}, k) \cap W(D \mapsto r, \mathcal{S}, k)$. The converse direction similarly holds.

In order to maintain the computation of the support, we use the bit vector $a$ of labels of windows from $T_{s}-k+1$ to $T_{e}-1$, which is a familiar technique for implementing the algorithm APRIORITID [1,2]. For the bit vector $a$ of the set $A$ of labels of windows, $i \in A$ if and only if $a_{i}=1$. Then, we can compute the intersection $A \cap B$ of two set of labels of windows as the bitwise logical product $a \odot b$ of two bit vectors $a$ of $A$ and $b$ of $B$. 


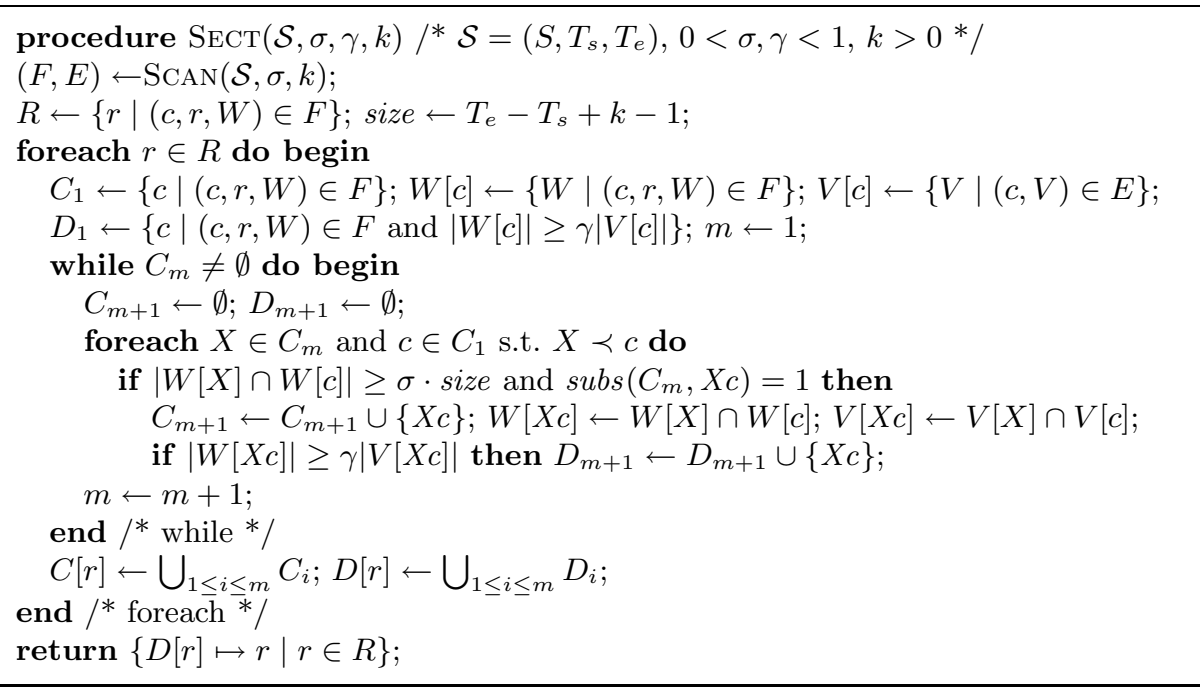

Fig. 3. The algorithm SECT.

Theorem 1. Let $\mathcal{S}$ be an event sequence on $\mathcal{E}$. Suppose that $M=\max \{|C| \mid$ $C \in C[r], r \in R\}$. Then, the algorithm SECT extracts all of the sectorial episodes that are frequent and confidential in $O\left(\left(l_{\mathcal{S}}+k\right)|\mathcal{E}|^{2 M+1}\right)$ time and $O\left(\left(l_{\mathcal{S}}+k\right)|\mathcal{E}|^{M}\right)$ space by traversing $\mathcal{S}$ just once.

Proof. By Lemma 2, the algorithm SCAN returns the set $F$ of triples $(c, r, W[c][r])$ such that $c \mapsto r$ is frequent and $W[c][r]=W(c \mapsto r, \mathcal{S}, k)$. Let $R$ be a set $\{r \mid(c, r, W) \in F\}$. For every $r \in R$, it is sufficient to show that $C[r]$ in SECT is the set of all causal types $X$ such that $X \mapsto r$ is frequent and confidential.

For $m \geq 1$, suppose that $C_{m}$ is the set of all causal types $X$ such that $|X|=m$ and $X \mapsto r$ is frequent. Then, consider the case $m+1$. Suppose that $X \in C_{m}$ and $c \in C_{1}$ such that $X \prec c$. Since $X \prec c$, it holds that $c \notin X$, so $|X c|=m+1$. Since $\operatorname{subs}\left(C_{m}, X c\right)=1$, every subset $Y \subset X c$ such that $|Y|=m$ is contained in $C_{m}$. By induction hypothesis, $Y \mapsto r$ is frequent. Note that $W[X]$ in the algorithm SECT denotes $W(X \mapsto r, \mathcal{S}, k)$. Then, by Lemma 3.2, it holds that $W[X] \cap W[c]=W(X \mapsto r, \mathcal{S}, k) \cap W(c \mapsto r, \mathcal{S}, k)=W(X c \mapsto r, \mathcal{S}, k)$. Hence, the condition $|W[X] \cap W[c]| \geq \sigma \cdot$ size means that $X c \mapsto r$ is frequent, so it holds that if $X c \in C_{m+1}$ then $X c \mapsto r$ is frequent.

Note that the algorithm SECT construct all causal types $Y$ such that $Y=X c$ and $Y \mapsto r$ is frequent from every $X \in C_{m}$ and $c \in C_{1}$. Hence, $C_{m+1}$ is the set of all causal types $Y$ such that $|Y|=m+1$ and $Y \mapsto r$ is frequent. Since $C[r]$ is the set $\bigcup_{1 \leq i \leq m} C_{i}$ such that $C_{i} \neq \emptyset(1 \leq i \leq m)$ and $C_{m+1}=\emptyset, C[r]$ in SECT is the set of all causal types $X$ such that $X \mapsto r$ is frequent.

Finally, since $V[X]$ in SECT denotes $W(X, \mathcal{S}, k)$ and by Lemma 3.1, the condition $|W[X]| \geq \gamma|V[X]|$ means that $X \mapsto r$ is confidential. Hence, every $D_{i}$ is the set of all causal types $X$ such that $|X|=i$ and $X \mapsto r$ is frequent and 
confidential, so $D[r]$ in SECT is the set of all causal types $X$ such that $X \mapsto r$ is frequent and confidential.

Next, consider the time and space complexity of SECT. For the inner foreachloop, it holds that $\left|C_{m}\right| \leq|\mathcal{E}|^{M}$. Since both $W[X]$ and $W[c]$ are regarded as bit vectors with length $l_{\mathcal{S}}+k-1$, the time to check whether or not $|W[X] \cap W[c]| \geq$ $\sigma \cdot$ size is $O\left(l_{\mathcal{S}}+k\right)$. Also we can determine whether $\operatorname{subs}\left(C_{m}, X c\right)=1$ or 0 by flipping 1 to 0 in the bit vector of $W[X c]$ and then by checking whether or not it is contained by $C_{m}$. Furthermore, since $V[e]$ is a bit vector with length $l_{\mathcal{S}}+k-1$, the time to check whether or not $|W[X c]| \geq \gamma|V[X c]|$ is $O\left(l_{\mathcal{S}}+k\right)$ time. Then, the time complexity of the inner foreach-loop is $O\left(l_{\mathcal{S}}+k+\left(l_{\mathcal{S}}+k\right)|\mathcal{E}|^{M}+l_{\mathcal{S}}+k\right)=$ $O\left(\left(l_{\mathcal{S}}+k\right)|\mathcal{E}|^{M}\right)$. Since the number of the outer and inner foreach-loop is at most $|\mathcal{E}|$ and $|\mathcal{E}|^{M}$, respectively, and by Lemma 2, the time complexity of SECT is $O\left(\left(l_{\mathcal{S}}+k\right)|\mathcal{E}|^{2}+\left(\left(l_{\mathcal{S}}+k\right)|\mathcal{E}|^{M}\right)|\mathcal{E}|^{M}|\mathcal{E}|\right)=O\left(\left(l_{\mathcal{S}}+k\right)|\mathcal{E}|^{2 M+1}\right)$. The space complexity is the space of bit vectors of $W[c][r]$ and $V[e]$, that is, $O\left(\left(l_{\mathcal{S}}+\right.\right.$ $\left.k)|\mathcal{E}|^{2}+\left(l_{\mathcal{S}}+k\right)|\mathcal{E}|^{M}+\left(l_{\mathcal{S}}+k\right)|\mathcal{E}|^{M-1}\right)=O\left(\left(l_{\mathcal{S}}+k\right)|\mathcal{E}|^{M}\right)$.

Finally, since the algorithm SECT does not traverse $\mathcal{S}$ and by Lemma 2, the algorithm SECT traverses $\mathcal{S}$ just once.

Example 2. Consider the event sequence $\mathcal{S}$ given in Figure 1. We give an running example of $\operatorname{SeCt}(\mathcal{S}, 0.5,0.6,3)$. Here, $W(\mathcal{S}, 3)=12$.

Consider $\operatorname{Scan}(\mathcal{S}, 0.5,3)$. Figure 4 (left) describes the sets $C, \operatorname{evtyp}(w(S, i, 3), i)$ and $R$ of event types at the end of foreach-loop for every $i(-1 \leq i \leq 8)$ in SCAN. Then, Figure 4 (right) describes the output of $\operatorname{ScAN}(\mathcal{S}, 0.5,3)$. Here, the column freq denotes the number of elements of $W[c][r]$ and the symbol $\bullet$, which is a label that $c \mapsto r$ is frequent.

Hence, $\operatorname{SCAN}(\mathcal{S}, 0.5,3)$ returns $(F, E)$, where $F$ and $E$ are the sets of triples and of pairs, respectively, as follows.

$$
\begin{aligned}
& F=\left\{\begin{array}{l}
(a, b, 1111000110),(c, b, 1111110110),(b, d, 1110011011), \\
(c, d, 1111010010),(a, e, 1101001110),(b, e, 1101101100), \\
(d, e, 1101101100),(b, c, 0111101100),(d, c, 0111101100)
\end{array}\right\}, \\
& E=\left\{\begin{array}{l}
(a, 111101111100),(b, 111111111110),(c, 111111111100), \\
(d, 111111111111),(e, 011111111110)
\end{array}\right\} .
\end{aligned}
$$

Next, consider $\operatorname{Sect}(\mathcal{S}, 0.5,0.6,3)$. From the set $F$, we obtain $R$ as $\{b, c, d, e\}$. In the following, we consider the constructions of $C[r]$ and $D[r]$, respectively, for every $r \in R$.

For $b \in R$, it holds that $C_{1}=\{a, c\}$. Since $W[a] \cap W[c]=1111000110 \odot$ $1111110110=1111000110$, it holds that $|W[a] \cap W[c]|=6$, so it holds that $C_{2}=\{a c\}$. Hence, it holds that $C[b]=\{a, c, a c\}$.

On the other hand, for $C_{1}=\{a, c\}$, it holds that $|W[a]|=6$ and $|V[a]|=10$, while $|W[c]|=8$ and $|V[c]|=9$. Then, it holds that $D_{1}=\{a, c\}$. Also since $V[a] \cap V[c]=111101111100 \odot 111111111100=111101111100$, it holds that $|V[a c]|=9$. Since $|W[a c]|=6$, it holds that $D_{2}=\{a c\}$, so $D[b]=\{a, c, a c\}$.

For $c \in R$, it holds that $C_{1}=\{b, d\}$. Since $W[b] \cap W[d]=0111101100 \odot$ $0111101100=0111101100$, it holds that $|W[b] \cap W[d]|=6$, so it holds that $C_{2}=\{b d\}$. Hence, it holds that $C[c]=\{b, d, b d\}$. 


\begin{tabular}{|c||c|c|c|}
\hline$i$ & $C$ & $\operatorname{evtyp}(w(S, i, 3), i)$ & $R$ \\
\hline-2 & $\emptyset$ & $\emptyset$ & $a b c d$ \\
-1 & $a b c d$ & $\emptyset$ & $b d e$ \\
0 & $a b c e d$ & $a c d$ & $a c$ \\
1 & $a b c e$ & $b e$ & $b d$ \\
2 & $a b c d$ & $a c$ & $c e$ \\
3 & $b e d e$ & $b d$ & $b$ \\
4 & $b c e$ & $c e$ & $a d$ \\
5 & $a b d$ & $b$ & $a c$ \\
6 & $a c d$ & $a d$ & $b e$ \\
7 & $b c e$ & $a c$ & $d$ \\
8 & $a b e$ & $b e$ & $\emptyset$ \\
\hline
\end{tabular}

\begin{tabular}{|c|c|c|c|c|c|c|c|}
\hline & \multicolumn{6}{|c|}{$\overline{W[c][r]}$} & \multirow[t]{2}{*}{ freq } \\
\hline & -1 & \begin{tabular}{l|l}
0 & 1
\end{tabular} & \begin{tabular}{|l|l|l|}
2 & 3 \\
\end{tabular} & 45 & 56 & & \\
\hline$a b$ & 1 & \begin{tabular}{l|l}
1 & 1
\end{tabular} & \begin{tabular}{|l|l|}
1 & 0 \\
\end{tabular} & 00 & & \begin{tabular}{l|l}
1 & 0
\end{tabular} & $6 \bullet$ \\
\hline$c \mid b$ & 1 & $1 \mid 1$ & \begin{tabular}{|l|l|l}
1 & 1 \\
\end{tabular} & $1 \mid 0$ & $0|1|$ & \begin{tabular}{l|l|l|l|l}
1 & 0
\end{tabular} & \\
\hline$d b$ & & $1 \mid \begin{array}{l}1 \\
1\end{array}$ & & $0 \mid 0$ & & $0 \mid 0$ & 5 \\
\hline$a \mid d$ & 1 & $1 \mid \begin{array}{l}1 \\
1\end{array}$ & \begin{tabular}{l|l|l|l|} 
& 0 \\
\end{tabular} & $0 \mid 0$ & $0|0|$ & $1 \mid 0$ & 5 \\
\hline$b \mid d$ & & $1 \mid 1$ & \begin{tabular}{l|l}
0 & 0
\end{tabular} & $|1| 1 \mid$ & $1|0|$ & \begin{tabular}{l|l|l}
1 & 1
\end{tabular} & 7 \\
\hline$c \mid d$ & 1 & $1 \mid \begin{array}{l}1 \\
1\end{array}$ & \begin{tabular}{l|l|l|l|}
1 & 0
\end{tabular} & $1 \mid 0$ & $0 \mid 0$ & $1 \mid 0$ & 6 \\
\hline$a \mid e$ & & $1 \mid 0$ & \begin{tabular}{l|l|l|}
1 & 0 \\
\end{tabular} & $|0| 1$ & & 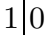 & 6 \\
\hline$b \mid e$ & 1 & $1 \mid 0$ & \begin{tabular}{|l|l|l}
1 & 1 \\
\end{tabular} & $\mid$\begin{tabular}{l|l}
0 & 1 \\
\end{tabular} & $1|1|$ & $0 \mid 0$ & 6 \\
\hline$c \mid e$ & 1 & $1 \mid 0$ & $1 \mid 0$ & $0 \mid 0$ & $0|1|$ & $1 \mid 0$ & 5 \\
\hline$d \mid e$ & 1 & $1 \mid 0$ & \begin{tabular}{|l|l|l|} 
& 1 & \\
\end{tabular} & \begin{tabular}{|l|l}
0 & 1
\end{tabular} & & \begin{tabular}{l|l}
0 & 0
\end{tabular} & 6 \\
\hline$b \mid a$ & 0 & $1 \mid 1$ & \begin{tabular}{l|l}
0 & 0
\end{tabular} & $|1| 1 \mid$ & $1|1|$ & $0 \mid 0$ & 5 \\
\hline$c \mid a$ & 0 & $1 \mid 0$ & $0 \mid 0$ & $1 \mid 0$ & & $0 \mid 0$ & 2 \\
\hline$d \mid a$ & 0 & $1 \mid \begin{array}{l}1 \\
1\end{array}$ & $0 \mid 0$ & $|0| 1$ & & $0 \mid 0$ & 4 \\
\hline$e \mid a$ & 0 & $1 \mid \begin{array}{l}1 \\
1\end{array}$ & $0 \mid 0$ & $|1| 0$ & 0 & \begin{tabular}{l|l}
0 & 0
\end{tabular} & 3 \\
\hline$a \mid c$ & 0 & $1 \mid 0$ & & \begin{tabular}{|l|l|l}
0 & 1
\end{tabular} & & $0 \mid 0$ & 4 \\
\hline$b \mid c$ & 0 & $1 \mid \begin{array}{l}1 \\
1\end{array}$ & \begin{tabular}{|l|l|l}
1 & 1
\end{tabular} & \begin{tabular}{|l|l|l}
0 & 1
\end{tabular} & $1|1|$ & \begin{tabular}{l|l}
0 & 0
\end{tabular} & \\
\hline$d \mid c$ & 0 & $1 \mid 1$ & & $\mid \begin{array}{lll}0 & 1\end{array}$ & $1 \mid 1$ | & $0 \mid 0$ & 6 \\
\hline e $c$ & 0 & $1 \mid \begin{array}{l}1 \\
1\end{array}$ & $0 \mid 0$ & $0 \mid 0$ & 0 & $0 \mid 0$ & 2 \\
\hline$e \mid b$ & 0 & \begin{tabular}{l|l}
0 & 1
\end{tabular} & \begin{tabular}{|l|l|l}
0 & 1
\end{tabular} & & 0 & $1 \mid 0$ & 5 \\
\hline$e d d$ & 0 & \begin{tabular}{l|l}
0 & 1 \\
\end{tabular} & \begin{tabular}{l|l}
0 & 0 \\
\end{tabular} & 10 & 0 & \begin{tabular}{l|l}
1 & 1 \\
\end{tabular} & 4 \\
\hline
\end{tabular}

Fig. 4. $C$, $\operatorname{evtyp}(w(S, i, 3), i)$ and $R$ at the end of foreach-loop (left) and $W[c][r]$ in SCAN (right).

On the other hand, for $C_{1}=\{b, d\}$, it holds that $|W[b]|=6$ and $|V[b]|=11$, while $|W[d]|=6$ and $|V[d]|=12$. Then, it holds that $D_{1}=\emptyset$. Also since $V[b] \cap V[d]=111111111110 \odot 111111111111=111101111110$, it holds that $|V[b d]|=11$. Since $|W[b d]|=6$, it holds that $D_{2}=\emptyset$, so $D[b]=\emptyset$.

For $d \in R$, it holds that $C_{1}=\{b, c\}$. Since $W[b] \cap W[c]=1110011011 \odot$ $1111010010=1110010010$, it holds that $|W[b] \cap W[d]|=5$, so it holds that $C_{2}=\emptyset$. Hence, it holds that $C[d]=\{b, c\}$.

On the other hand, for $C_{1}=\{b, c\}$, it holds that $|W[b]|=7$ and $|V[b]|=$ 11 , while $|W[c]|=6$ and $|V[c]|=10$. Then, it holds that $D_{1}=\{b, c\}$. Also since $V[b] \cap V[c]=111111111110 \odot 111111111100=111101111100$, it holds that $|V[b c]|=10$. Since $|W[b c]|=5$, it holds that $D_{2}=\emptyset$, so $D[d]=\{b, c\}$.

For $e \in R$, it holds that $C_{1}=\{a, b, d\}$. Then, the following statement holds.

$$
\begin{aligned}
& W[a] \cap W[b]=1101001110 \odot 1101101100=1101001100, \\
& W[a] \cap W[d]=1101001110 \odot 1101101100=1101001100, \\
& W[b] \cap W[d]=1101101100 \odot 1101101100=1101101100 .
\end{aligned}
$$

Then, it holds that $|W[a] \cap W[b]|=5,|W[a] \cap W[d]|=5$ and $|W[b] \cap W[d]|=6$. Hence, it holds that $C_{2}=\{b d\}$, so it holds that $C[e]=\{a, b, d, b d\}$.

On the other hand, for $C_{1}=\{a, b, d\}$, it holds that $|W[a]|=6,|V[a]|=9$, $|W[b]|=6,|V[b]|=11,|W[d]|=6$ and $|V[d]|=12$. Then, it holds that $D_{1}=\{a\}$. 
Also since $|V[a] \cap V[b]|=9,|V[a] \cap V[d]|=9$ and $|V[b] \cap V[d]|=11$, it holds that $D_{2}=\emptyset$, so $D[e]=\{a\}$.

As the result, the algorithm $\operatorname{Sect}(\mathcal{S}, 0.5,0.6,3)$ returns the following frequent sectorial episodes, where ones with bold faces are frequent and confidential.

$$
\begin{array}{rlrlrl}
\mathbf{a} & \mapsto \mathbf{b} & \mathbf{c} \mapsto \mathbf{b} & \mathbf{a c} \mapsto \mathbf{b} & \\
b & \mapsto c & d \mapsto c & b d \mapsto c & \\
\mathbf{b} \mapsto \mathbf{d} & \mathbf{c} \mapsto \mathbf{d} & & \\
\mathbf{a} \mapsto \mathbf{e} & b \mapsto e & d \mapsto e & b d \mapsto e
\end{array}
$$

\section{Empirical Results}

In this section, by applying the algorithm SECT to bacterial culture data, which are complete data in [8] from 1995 to 1998, we extract sectorial episodes concerned with drug-resistant change.

First, we fix the width of windows as 30 days. Since the database contains the patient information not related to drug-resistant change such as date, gender, ward and engineer [8], it is necessary to focus the specified attributes. Then, we select the attributes age, department, sample, fever, catheter, tracheo, intubation, drainage, WBC (white blood cell) count, medication, Urea-WBC, UreaNitocide, Urea-Occultblood, Urea-Protein, the total amount of bacteria, the detected bacterium, and the sensitivity of antibiotics as the causal types, and the sensitivity of antibiotics as the resulting type.

From the medical viewpoint, in order to extract sectorial episodes concerned with drug-resistant change, it is necessary to extract them based on the same detected bacterium and the same sample. Hence, in this paper, we divide the database into pages for the detected bacterium and the sample described as Figure 5 in whole 44 attributes. The column "patients" denotes the number of different patients consisting of more than two records in a page, and the column "max." denotes the maximum number of records for patients in a page. Here, the detected bacteria are Staphylococci (bac1), Enteric bacteria (bac7), glucose-nonfermentative gram-negative bacteria (bac8) and Anaerobes (bac11), and the samples are catheter/others (spl1), urinary and genital organs (spl3) and respiratory organs (spl5). Then, by connecting all records in a page into one event sequence such that the span between all records for one patient and ones for other patient is at least 30 days (the width of windows), we apply the algorithm SECT to it.

For the minimum support $\sigma=0.15$ and the minimum confidence $\gamma=0.8$, the column "episodes" in Figure 5 describes the number of the extracted sectorial episodes. Furthermore, we focus on the extracted sectorial episode $C \mapsto r$ such that, for antibiotics Ant, $C$ contains "Ant=S (susceptibility)" and $r$ is "Ant=R (resistant)." In the column "antibiotics" in Figure 5, Ant $(n)$ denotes that $n$ is the number of extracted sectorial episodes of the form $C \mapsto($ Ant=R $)$ such that $(\mathrm{Ant}=\mathrm{S}) \in C$. Here, antibiotics are benzilpenicillin (PcB), augmentin (Aug), anti-pseudomonas penicillin (PcAP), 1st generation cephems (Cep1), 2nd generation cephems (Cep2), 3rd generation cephems (Cep3), anti-pseudomonas cephems 
(CepAP), aminoglycosides (AG), macrolides (ML), tetracyclines (TC), carbapenems (CBP), and RFP/FOM (RFPFOM).

\begin{tabular}{|c|c|c|c|c|c|}
\hline bacterium & sample & patients & $\max$. & episodes & antibiotics \\
\hline \multirow[t]{2}{*}{ bac1 } & spl1 & 296 & 34 & 2668 & $\operatorname{RFPFOM}(8), \mathrm{TC}(1)$ \\
\hline & spl5 & 319 & 19 & 27174 & $\operatorname{CBP}(225), \operatorname{RFPFOM}(148), \operatorname{ML}(1), \mathrm{TC}(1)$ \\
\hline \multirow[t]{2}{*}{ bac7 } & spl3 & 131 & 10 & 21957 & $\begin{array}{l}\text { Cep3(340), AG(323), CepAP(79), TC(16), } \\
\text { Cep2(5), Cep1(3), Aug }(2)\end{array}$ \\
\hline & spl5 & 301 & 21 & 76951 & $\begin{array}{l}\operatorname{CBP}(1334), \operatorname{CepAP}(1158), \operatorname{Cep} 3(184), \\
\operatorname{PcAP}(128), \operatorname{TC}(98), \operatorname{Aug}(2), \operatorname{Cep} 1(2), \\
\text { Cep2 }(2)\end{array}$ \\
\hline \multirow[t]{2}{*}{ bac8 } & spl1 & 81 & 7 & 8127 & $\operatorname{AG}(1), \operatorname{CepAP}(1), \operatorname{PcAP}(1)$ \\
\hline & spl5 & 296 & 21 & 37938 & CepAP $(40), \operatorname{AG}(8), \operatorname{CBP}(1)$ \\
\hline bac11 & spl1 & 208 & 22 & 16720 & $\begin{array}{l}\operatorname{CBP}(304), \operatorname{Cep} 2(81), \operatorname{Cep} 3(81), \operatorname{Cep} 1(1) \\
\operatorname{ML}(1), \operatorname{PcAP}(1), \operatorname{PcB}(1)\end{array}$ \\
\hline
\end{tabular}

Fig. 5. The pages for the detected bacterium and the sample from bacterial culture data, the number of extracted sectorial episodes under $\sigma=0.15$ and $\gamma=0.8$, and the antibiotics, where $\operatorname{Ant}(n)$ denotes that $n$ is the number of extracted sectorial episodes of the form $C \mapsto($ Ant=R) such that $($ Ant=S $) \in C$.

Figure 5 means that different sectorial episodes representing drug-resistant change are extracted for every detected bacterium and sample. For (bac1,sp15), (bac7,sp15) and (bac11,spl1), the drug-resistant change for CBP occurs in the extracted episodes. In particular, for (bac7,spl5), the drug-resistant change CepAP also occurs in the extracted episodes. On the other hand, for (bac7,spl3), the drug-resistant change Cep3 and AG occurs in the extracted episodes.

\section{Conclusion}

In this paper, we have newly introduced the sectorial episode together with the parallel episode [5]. Then, we have designed the algorithm SECT to extract all of the sectorial episodes that are frequent and confidential. Finally, we have applied the algorithm SECT to bacterial culture data, and extracted sectorial episodes representing drug-resistant change.

Since the number of extracted sectorial episodes in Section 4 is large, it is a future work to introduce the concept of closed sectorial episodes like as closed sequential patterns $[9,10]$, in order to reduce the number of extracted episodes.

In Section 4, we have treated the pages for patients as one event sequence such that every page is far from at least the width of windows. Then, it is a future work to introduce another frequency measure like as the frequency of pages. Also it is a future work to apply our algorithm to another time-related data and extract sectorial episodes from them. 
As stated in Section 1, since a sectorial episode has been captured as the direct precedent-subsequent relationship of events, it is just a candidate of causality in database ( $c f$. [11]). Hence, it is an important future work to incorporate such causality with our sectorial episodes and to design the algorithm to extract sectorial episodes concerned with causality.

\section{References}

1. R. Agrawal, H. Mannila, R. Srikant, H. Toivonen, A. I. Verkamo: Fast discovery of association rules, in U. M. Fayyed, G. Piatetsky-Shapiro, P. Smyth, R. Uthurusamy (eds.): Advances in Knowledge Discovery and Data Mining, AAAI/MIT Press, 307-328, 1996.

2. R. Agrawal, R. Srikant: Fast algorithms for mining association rules in large databases, Proc. 20th VLDB, 487-499, 1994.

3. R. Agrawal, R. Srikant: Mining sequential patterns, Proc. 11th ICDE, 3-14, 1995.

4. C. Bettini, S. Wang, S. Jajodia, J.-L. Lin: Discovering frequent event patterns with multiple granularities in time sequences, IEEE Trans. Knowledge and Data Engineering 10, 222-237, 1998.

5. H. Mannila, H. Toivonen, A. I. Verkamo: Discovery of frequent episodes in event sequences, Data Mining and Knowledge Discovery 1, 259-289, 1997.

6. J. Pei, J. Han, B. Mortazavi-Asi, J. Wang, H. Pinto, Q. Chen, U. Dayal, M.C. Hsu: Mining sequential patterns by pattern-growth: The PrefixSpan approach, IEEE Trans. Knowledge and Data Engineering 16, 1-17, 2004.

7. R. Srikant, R. Agrawal: Mining sequential patterns: Generalizations and performance improvements, Proc. 5th EDBT, 3-17, 1996.

8. S. Tsumoto: Guide to the bacteriological examination data set, in E. Suzuki (ed.): Proc. International Workshop of KDD Challenge on Real-World Data (KDD Challenge 2000), 8-12, 2000.

9. J. Wang, J. Han: BIDE: Efficient mining of frequent closed sequences, Proc. 20th ICDE, 2004.

10. X. Yan, J. Han, R. Afshar: CloSpan: Mining closed sequential patterns in large datasets, Proc. 3rd SDM, 2003.

11. C. Zhang, S. Zhang: Association rule mining, Springer-Verlag, 2002. 